\title{
QUEEN'S
UNIVERSITY
BELFAST
}

\section{Curl maps in nanowires}

McCooey, C., Todorov, T., \& Dundas, D. (2020). Curl maps in nanowires. Physical Review B, 102, 115403-1 115403-11. [115403]. https://doi.org/10.1103/PhysRevB.102.115403

\author{
Published in: \\ Physical Review B
}

\section{Document Version:}

Peer reviewed version

Queen's University Belfast - Research Portal:

Link to publication record in Queen's University Belfast Research Portal

\section{Publisher rights}

Copyright200 American Physical Society. This work is made available online in accordance with the publisher's policies. Please refer to any applicable terms of use of the publisher.

\section{General rights}

Copyright for the publications made accessible via the Queen's University Belfast Research Portal is retained by the author(s) and / or other copyright owners and it is a condition of accessing these publications that users recognise and abide by the legal requirements associated with these rights.

\section{Take down policy}

The Research Portal is Queen's institutional repository that provides access to Queen's research output. Every effort has been made to ensure that content in the Research Portal does not infringe any person's rights, or applicable UK laws. If you discover content in the Research Portal that you believe breaches copyright or violates any law, please contact openaccess@qub.ac.uk. 


\title{
Curl maps in nanowires
}

\section{Christopher McCooey, ${ }^{*}$ Tchavdar N. Todorov, and Daniel Dundas}

Atomistic Simulation Centre,

School of Mathematics and Physics,

Queen's University Belfast,

Belfast BT7 1NN, UK

(Dated: September 29, 2020)

\begin{abstract}
Nonconservative current-induced forces (NCIF) in nanowires have attracted attention both as a failure mechanism and as a driver for atomic-scale motors. Here we investigate the view that the nonconservative character of the electron wind force is an intrinsic property of the electron flow. To this end we consider the curl of the force on a noninvasive test scatterer immersed in the electron current. Using a free-electron Hamiltonian, we find nonzero curls at the opening of conductance channels due to variations in the local density of states. Current vortices are observed in these simulations, and one would naively expect them to give rise to large curls. However, this is not always the case. We find two types, low- and high-curl current vortices. Bouncy patterns, the 'snaking' of the current flow through the wire, are a prominent effect that gives rise to large curls. Ultimately NCIF reduce the stability of nanowires and therefore crucial to study their origin to manage their effects. This approach enhances our understanding of NCIF by going beyond forces on individual nuclei.
\end{abstract}




\section{INTRODUCTION}

Over the past 50 years, the size of electronic devices has decreased in accordance with Moore's law $^{1}$. A key challenge has been improving their structural stability and suitability for applications in industry ${ }^{2}$. Electromigration is the atomic diffusion and rearrangements of atoms in a conductor and was initially thought of as a thermally activated process ${ }^{3}$. However, Sorbello proposed a thought experiment, illustrated in Fig. 1, to show that the underlying driving electron wind force (EWF) is nonconservative ${ }^{4}$. This nonconservative current-induced force (NCIF) can do net work on the nuclei around closed paths. The resultant energy transfer to the atomic motion can itself become an activation mechanism for electromigration.

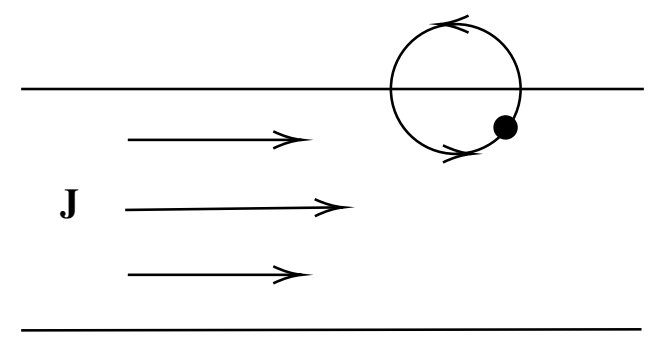

FIG. 1. Sorbello's thought experiment: a scatterer (black dot), immersed in current density, J, follows a closed path. Inside the wire the scatterer experiences the EWF. Outside the wire there is no force on the scatterer. In this way the EWF does net work on the scatterer around this closed path.

The EWF is only one of the several forces experienced by nuclei in atomic wires. Other contributions include velocity-dependent forces and the force noise, responsible for inelastic scattering and Joule heating ${ }^{5-8}$. The EWF results fundamentally from the mean momentum transfer in elastic electron scattering by nuclei and forms the focus of the present work.

An initial debate whether this current-induced force ${ }^{9}$ could be nonconservative was finally resolved by explicitly evaluating its curl and showing it to be non-zero even under ideal steady-state conditions ${ }^{10}$. It has since received fresh attention due to its nonconservative character ${ }^{10-17}$. The EWF has been shown to drive the motion of the nuclei in a manner similar to a waterwheel being driven by a river ${ }^{10}$, opening up the possibility of using an applied bias to drive and control the motion of an atomic motor ${ }^{10,14,15,18}$. As a failure mechanism, the EWF is potentially fatal. The current densities in nanowires can be many orders of magnitude larger compared to their Ohmic counterparts. These large current densities can result in substantial NCIF ${ }^{19,20}$ and violent dynamics of the nuclei. Under certain conditions, the nonconservative EWF could be a more powerful 
activation mechanism of electromigration than Joule heating ${ }^{21}$.

The concept of NCIF is more general than forces on nuclei. By considering the force on a noninvasive test scatterer immersed at an arbitrary point in the steady-state electron current of a wire, we obtain a quantity that is an intrinsic property of the flow ${ }^{22}$. In this paper, we adopt this view to investigate how the curl of the EWF on a test particle behaves throughout the interior of nanoscale wires. We present our results in the form of curl maps, analogous to power maps for currentcarrying nanostructures ${ }^{23}$. On the boundary between the wire and the vacuum, it is easy to demonstrate the presence of non-zero curls from a pen and paper inspection of the current density ${ }^{12}$. However, the behaviour of the curl within the wire is not so readily understood from the current density alone. In these regions, the curl is determined by the interplay between the local density of states and current density. This paper offers fresh insight into the behaviour of the curl of the EWF. By considering its geometry- and energy-dependence, we provide a way in principle for this behaviour to be controlled. Extending our investigation from the sample into the adjoining leads reveals exciting behaviour. We have observed bouncy patterns in the current density along the leads and reconstriction of the current flow, in a quasi-periodic fashion. These effects are potentially a disastrous cause of electromigration in the leads.

The rest of the paper is organised as follows. Sec. II presents the method used to calculate the electronic properties of the systems of interest, Sec. III discusses our findings in three systems with methods to control the formation of these large curls. Finally we give a discussion and concluding comments in Sec. IV and Sec. V.

\section{METHOD}

We investigate a current-carrying conductor using a free-electron Hamiltonian. Non-interacting electrons are considered throughout. The current flows through a sample connected to two leads, under an applied electrochemical bias, in the absence of magnetic fields. To gain insight into the behaviour of the curl of the EWF, described in Sec. II D, we will neglect the velocity-dependent forces $^{22}$, and consider ideal steady-state conditions. The quantities needed to investigate the curl - the current density and the local density of states - will be obtained with the aid of the Landauer picture of transport expressed in terms of Green's functions. 


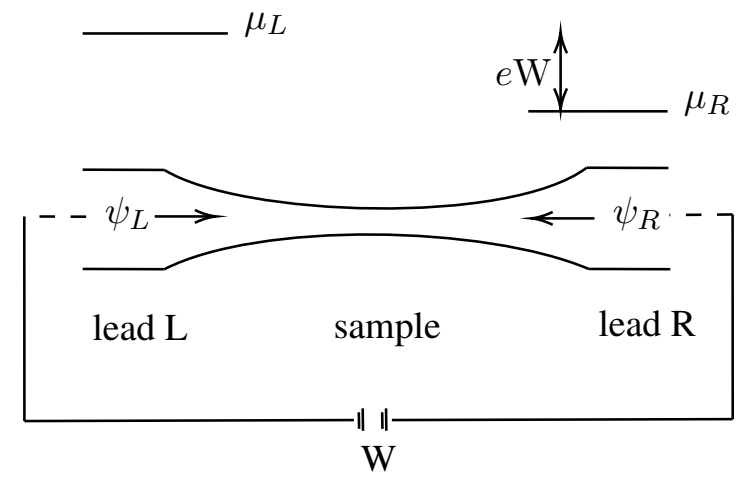

FIG. 2. Landauer picture of electronic transport: the system can have an arbitrary geometry and composition. Details are discussed in the text.

\section{A. Landauer Picture of Transport}

The conductor of interest is connected to, in this case, two semi-infinite perfect leads as shown in Fig. 2. The left and right lead are connected to particle reservoirs with electrochemical potentials $\mu_{L}$ and $\mu_{R}$, respectively. $e W=\mu_{L}-\mu_{R}$ is the applied bias. A group of right-travelling states $\left\{\left|\psi_{L}\right\rangle\right\}$ with energies $\left\{E_{L}\right\}$ are partially transmitted into lead $R$ and partially reflected back. The converse happens for the left-travelling group of states $\left\{\left|\psi_{R}\right\rangle\right\}$ with energies $\left\{E_{R}\right\}$. The partial density of states operators $\hat{D}_{L}(E)$ and $\hat{D}_{R}(E)$ are defined by

$$
\begin{aligned}
& \hat{D}_{L}(E)=\sum_{L}\left|\psi_{L}\right\rangle \delta\left(E-E_{L}\right)\left\langle\psi_{L}\right| \\
& \hat{D}_{R}(E)=\sum_{R}\left|\psi_{R}\right\rangle \delta\left(E-E_{R}\right)\left\langle\psi_{R}\right| .
\end{aligned}
$$

\section{B. Discretising the Hamiltonian}

A finite difference approximation is employed to discretise space and approximate the Hamiltonian of the system. This discretisation allows the properties of the current-carrying electronic system to be probed throughout a given region of interest. The Hamiltonian, $\hat{H}$, for the system is given by

$$
\hat{H}=-\frac{\hbar^{2}}{2 m} \nabla^{2}+U(\mathbf{r})
$$

We will consider only the $2 \mathrm{D}$ case with a Cartesian coordinate system with a uniform grid spacing a. Let position on the computational mesh be labelled with index $i$, and let $\psi_{i}$ and $U_{i}$ be the statevector amplitude and potential at the mesh point $i$ respectively. The time-independent Schrödinger 
equation then takes the matrix form

$$
\sum_{j} H_{i j} \psi_{j}=E \psi_{i}
$$

where

$$
\begin{aligned}
& H_{i i}=\frac{2 \hbar^{2}}{m a^{2}}+U_{i} \equiv \mathcal{E}_{i}, \\
& H_{i j}=-\frac{\hbar^{2}}{2 m a^{2}}=\gamma \forall \text { nearest neighbours, } \\
& H_{i j}=0 \text { otherwise. }
\end{aligned}
$$

This discretised Hamiltonian is physically distinct from a nearest-neighbour orthogonal singleorbital tight-binding model but the two are similar mathematically ${ }^{24-26}$.

\section{Dyson equation \& Green's Function}

The retarded surface Green's function for the left and right leads, $\hat{g}_{L}^{+}$and $\hat{g}_{R}^{+}$, are equal and analytically known ${ }^{26,27}$. The element connecting mesh point $i$ to $j$ is given by

$$
\left[g_{L}^{+}(E)\right]_{i j}=\frac{2}{N+1} \sum_{p=1}^{N} \sin \left(\frac{p \pi i}{N+1}\right) \sin \left(\frac{p \pi j}{N+1}\right) g_{0}(E, p)
$$

where $N$ is the number of grid points in the cross-section of the lead and

$$
\begin{aligned}
& g_{0}(E, p)=\frac{E-E(p) \pm \sqrt{[E-E(p)]^{2}-4 \gamma^{2}}}{2 \gamma^{2}} \\
& E(p)=-2|\gamma| \cos \left(\frac{p \pi}{N+1}\right)
\end{aligned}
$$

where $p$ labels the channels in the leads. We choose the solution in Eqn. (7) with the minus sign in front of the square root if the expression under the square root is negative, while if this expression is positive, we choose the solution with the minus if $E-E(p)>0$ and the solution with the plus sign if $E-E(p)<0^{26,27}$. The Green's function for the disconnected sample is given by

$$
\begin{aligned}
{\left[g_{s}^{+}(E)\right]_{i i} } & =\frac{1}{E-\mathcal{E}_{i}+\mathrm{i} \epsilon} \\
{\left[g_{s}^{+}(E)\right]_{i j} } & =0 \forall i \neq j
\end{aligned}
$$

where $\mathrm{i} \epsilon$ is a small complex energy. The unperturbed Green's function for the system, $\hat{g}^{+}$, is given by $\hat{g}^{+}(E)=\hat{g}_{L}^{+}(E)+\hat{g}_{R}^{+}(E)+\hat{g}_{s}^{+}(E)$. The potential connecting the sample and leads together is 


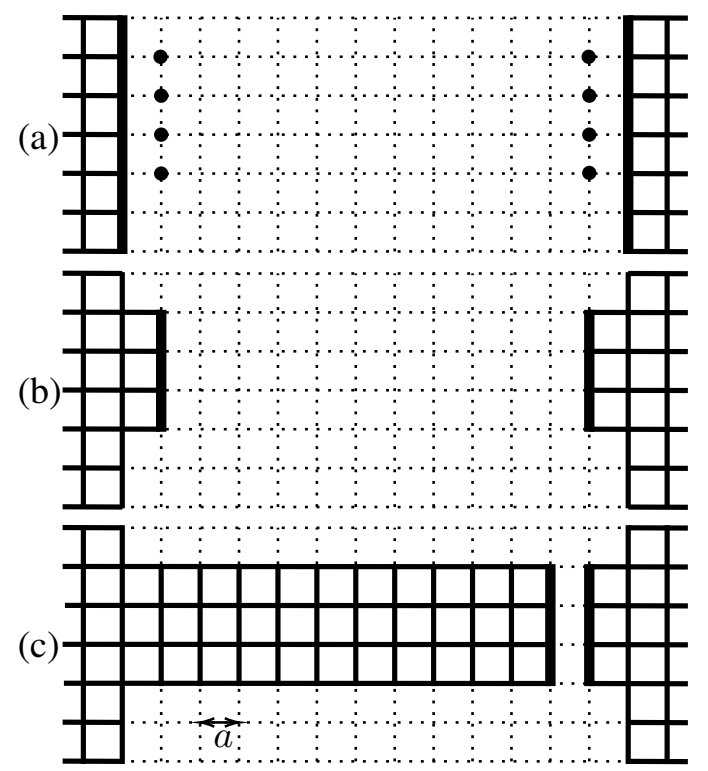

FIG. 3. Schematic of the layer by layer growth procedure with $a$ representing the grid spacing of the mesh. (a) The Green's function is known on the surface of the leads shown with the thick black line and at the mesh points (black dots) being bonded to the leads. (b) Once the potential is turned on and the Dyson equation solved, the new surface Green's function is found. (c) This process is repeated both on the left and right lead until the two are connected together.

given by

$$
V_{i j}= \begin{cases}\gamma & \forall i \text { and } j \text { nearest neighbours in the sample, } \\ \gamma & \forall i \text { and } j \text { connecting the lead to the sample, } \\ 0 & \text { otherwise. }\end{cases}
$$

The Green's function for the bonded system, $\hat{G}^{+}$, can then be found by solving the Dyson equation in one step, but a more efficient way is the layer by layer growth method ${ }^{26,27}$ shown in Fig. 3. At each growth stage, a layer is added onto the left and right lead and the Dyson equation is solved

$$
\left(\hat{I}-\hat{g}^{+} \hat{V}\right) \hat{G}^{+}=\hat{g}^{+}
$$

This process calculates the new Green's function on the surface of the lead. Once the two leads meet, the Dyson equation is solved again, and the Green's function for the connected system is found for the desired two layers. This process is carried out along the sample, building up the Green's function for the coupled system. The geometry of the wire can be altered by setting the onsite energy, $\mathcal{E}_{i}$, of chosen mesh points to inaccessible levels. Instabilities in calculating the 
Green's function can occur when the Green's function is singular. This is avoided by moving the electron energy off the real axis through a small complex energy, $\mathrm{i} \epsilon$. This complex energy needs to be large enough such that numerical truncation does not put the energy back on the real axis but also small enough to avoid current leakage ${ }^{26}$.

To express the density of states in terms of the Green's function, we introduce an arbitrary cut, $S$, across the coupled system ${ }^{24}$. This separates the system into two parts $A$ and $B$. We define $\hat{P}_{A}$ as the projection operator for part $A$ and $\hat{P}_{B}$ as the projection operator for part $B$. We can now write the density of states emanating from left and right lead ${ }^{24}$ as

$$
\begin{aligned}
& 2 \pi \mathrm{i} \hat{D}_{L}=\hat{P}_{A} \hat{G}^{-}-\hat{G}^{+} \hat{P}_{A}+\hat{G}^{+}\left(\hat{V} \hat{P}_{A}-\hat{P}_{A} \hat{V}\right) \hat{G}^{-} \\
& 2 \pi \mathrm{i} \hat{D}_{R}=\hat{P}_{B} \hat{G}^{-}-\hat{G}^{+} \hat{P}_{B}+\hat{G}^{+}\left(\hat{V} \hat{P}_{B}-\hat{P}_{B} \hat{V}\right) \hat{G}^{-}
\end{aligned}
$$

where $\hat{G}^{-}$is the advanced Green's function and given by $\hat{G}^{-}=\hat{G}^{+\dagger}$. The full density of states, $\hat{D}(E)$, for the lead-sample-lead system is given by

$$
\hat{D}=\hat{D}_{L}+\hat{D}_{R}
$$

To obtain the local density of states (LDOS) per unit energy interval, per unit area, the diagonal elements of $\hat{D}$ in the real-space basis need to be divided by $a^{2}$. The particle current density, $\mathbf{J}_{i}$, at a point $i$ needs to be constructed next ${ }^{24}$. The bond current from site $i$ to site $j$ is given by

$$
J_{i j}=\frac{4 e W \gamma}{\hbar} \operatorname{Im}\left[D_{L}(E)_{i j}\right]
$$

However, we need the flow at site $i$. This can be calculated by averaging the bond current in and out of a site to build up a vector, $\mathbf{J}_{i}$, for the current density at point $i$. To normalise the current density, $\mathbf{J}_{i}$ needs to be divided by $a$.

\section{Curl of the Electron Wind Force}

The curl, $\mathbf{C}(\mathbf{R})$, of the EWF at a general point $\mathbf{R}$ can be defined as the curl of the force on a noninvasive test scatterer immersed in the current flow at that point. Let the potential, $v$, of the probe be $v(\mathbf{R})=\zeta \delta(\mathbf{r}-\mathbf{R})$ where $\zeta$ is the coupling constant. We shall neglect the influence of phonons and operate at length scales below the electron inelastic mean free path. To lowest order in $\zeta$, in the low bias limit, the curl of the EWF on the test scatterer is given by

$$
\mathbf{C}(\mathbf{R})=\frac{2 \pi m \zeta^{2}}{\hbar}\left\{\nabla_{\mathbf{r}} \times[D(\mathbf{r}, E) \mathbf{J}(\mathbf{r})]\right\}_{\mathbf{r}=\mathbf{R}}
$$


where $D(\mathbf{r}, E)$ is the LDOS at the Fermi level, $E$, and $\mathbf{J}(\mathbf{r})$ is the current density in the absence of the test scatterer ${ }^{22}$. Ignoring prefactors, the curl in Eqn. 16 can be expanded out into the two contributions $\left[\nabla_{\mathbf{r}} D(\mathbf{r}, E)\right]_{\mathbf{r}=\mathbf{R}} \times \mathbf{J}(\mathbf{R})$ and $D(\mathbf{R}, E)\left[\nabla_{\mathbf{r}} \times \mathbf{J}(\mathbf{r})\right]_{\mathbf{r}=\mathbf{R}}$. The latter is arguably the more intuitive; the former captures the effect of spatial variations in the LDOS.

\section{E. Convergence}

In the 2D systems explored in this paper we will be considering the $z$-component of the curl. Care needs to be taken when employing a mesh to test the convergence of the calculation. To conserve the physical dimensions of the wire, the mesh must be constructed according to Fig. 4. Three different methods were used to give a measure of the convergence for each system studied. The first and simplest was to compare the conductance as a function of energy for different mesh sizes. The conductance quickly converges for decreasing mesh size for each system. However, it falls short in answering how the curl, the main quantity of interest, behaves with mesh size. A more rigorous method involves calculating the fractional percentage difference, $L_{a}(E)$,

$$
L_{a}(E)=\frac{1}{M} \sum_{i \in A}\left|\frac{C_{i}^{a}-C_{i}^{\frac{a}{2}}}{C_{i}^{a}+C_{i}^{\frac{a}{2}}}\right|
$$

where $A$ are the elements common to both meshes and with a magnitude larger than $1 \%$ of the maximum curl at each energy, $M$ is the number of elements in $A$ and $C_{i}^{a}$ is the curl element $i$ from mesh $a$. Neglecting elements with a magnitude less than the $1 \%$ cutoff helps avoid including entries with near-zero curl. The curl was found to converge slowly with decreasing mesh size. This is in part due to the ability of the curl to change sign which can allow the denominator in Eqn. 17 to tend to zero, the compounding of error from the current density and LDOS and from using finite differences to evaluate Eqn. 16. At energies where the definition of $L_{a}(E)$ proved particularly problematic, a side-by-side visual comparison was used to judge the convergence. More information on the convergence of the curl and plots of the conductance, $L_{a}(E)$ and visual comparison are provided in Appendix A.

\section{RESULTS}

Simple systems, namely the perfect wire, the $90^{\circ}$ S-bend shown in Fig. 5 and the constriction shown in Fig. 6 were investigated. These systems were chosen to aid the understanding of the 


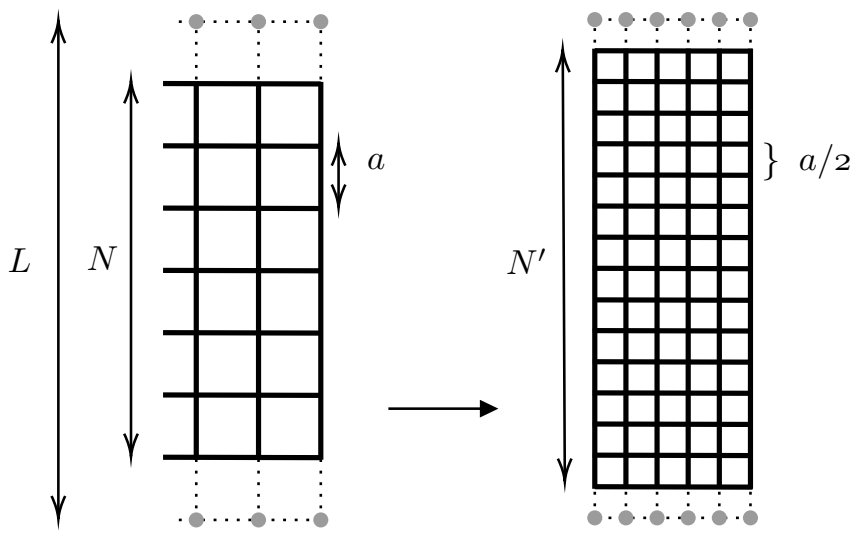

FIG. 4. When reducing the mesh size of the computational grids care needs to be taken to ensure the physical dimensions of the system are conserved. The ghost sites (grey dots) represent the length, $L$, between the hard walls. Ghost sites are used in the formulation of the Green's function, and the boundary of the wire must not be confused with the edge of the mesh. Here the mesh size goes from $a$ to $a / 2$. The number of mesh points in the cross-section goes as $N^{\prime}=2 N+1$ where $N$ is the number of mesh points in the larger mesh, thereby conserving the cross-sectional length $L=(N+1) a=\left(N^{\prime}+1\right) a / 2$.

behaviour of the curl. In each system, hard walls were used to mark the boundary of the wire. The Fermi energy was measured relative to the bottom of the conduction band, $-4|\gamma|$. The perfect wire, Sec. III A, was investigated first and gave a yardstick for other systems. This yardstick is a natural one as it rests on the same foundation as Sorbello's thought experiment. The $90^{\circ}$ S-bend, Sec. III B, and the constriction, Sec. III C, both showed exciting features in the curl.

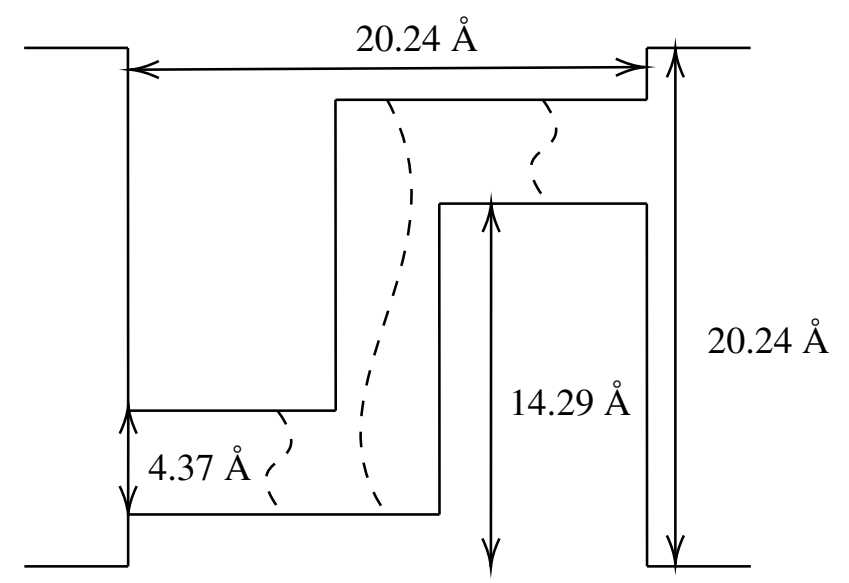

FIG. 5. Schematic: $90^{\circ}$ S-bend. Large curls are observed when standing waves in the LDOS (dashed line) are present in the lower-left, upper-right and middle section. The onsite energy within the wire is set to $0 \mathrm{eV}$ and outside is set to $54400 \mathrm{eV}$. 


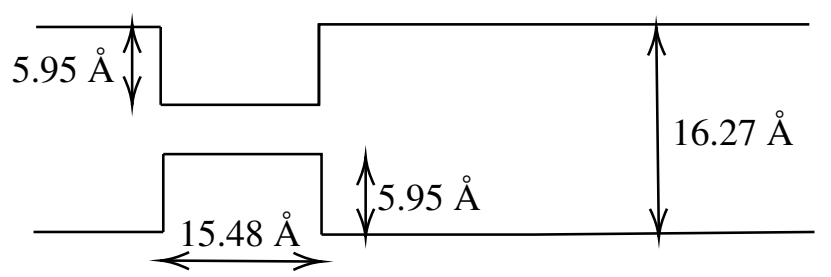

FIG. 6. Schematic: Constriction connected to perfect leads. Large curls are found in the constriction when standing waves in the LDOS are formed in the cross-section. The onsite energy within the wire is set to $0 \mathrm{eV}$ and outside is set to $54400 \mathrm{eV}$.

\section{A. Perfect Wire}

The first system investigated was a perfect wire of width $L=16.27 \AA$. The perfect wire is solvable analytically and can serve as a benchmark to test the method. Large curls are observed at the surface and within the body of the wire at energies where a conductance channel opens. In Fig. 7, standing waves in the LDOS have formed in the cross-section, in conjunction with the current density, giving rise to large curls within the wire and even larger ones near the surface. Large curls due to the opening of conductance channels are observed in all systems studied. The opening of a conductance channel can at least, in theory, be controlled geometrically by altering the crosssection of the sample, thereby mitigating this effect.

\section{B. $90^{\circ}$ S-bend}

The geometry of the S-bend, shown in Fig. 5, has point inversion symmetry which can be used to test the calculation. Like the perfect wire in Sec. III A, large curls are observed at the opening of conductance channels and when LDOS standing waves are formed in the wire.

Bouncy patterns are regions where the current 'snakes' along the wire, causing large curls in its wake. In Fig. 8, there is a bouncy pattern with a high curl in the lower-left and upper-right sections of the wire. These patterns are formed from the mixing of different conducting modes open in the $90^{\circ} \mathrm{S}$-bend. An argument for this can be found in Appendix B. At this energy, there are two conductance channels open in the sample. The formation of bouncy patterns can be controlled by reducing the cross-section so that only one conductance channel can open in the 
sample. Bouncy patterns can be controlled in theory by rounding the corners ${ }^{28}$ or by increasing the radius of curvature ${ }^{29}$. This helps reduce mode-mixing at energies with multi-mode transport.

The presence of current vortices allows for the circulation about a vortex centre to be many times larger than the net current across the sample. Current vortices are formed in regions where closed classical paths can be constructed ${ }^{30,31}$. Vortices can occur as a lone pair, shown in Fig. 8 and Fig. 9, or in larger groups, as in Fig. 10. Two types of vortices have been observed, high- and low-curl. In Fig. 9 we can see an example of a large curl vortex located in the corners of the wire. Non-zero curls are expected with the formation of current vortices. Large curls in the presence of current vortices are not, however, the norm. Current vortices alone cannot give rise to large curls in the absence of a sizeable LDOS and current flow. Evidence of this can be seen in Fig. 10, a triplet vortex. Despite the large internal local currents relative to the net current, they are tiny relative to the currents in Fig. 9 which accounts for the low-curl in Fig. 10.

\section{Constriction}

The constriction, shown in Fig. 6, is geometrically simpler than the $90^{\circ} \mathrm{S}$-bend and has symmetry about the horizontal axis of the wire. We have observed large curls at the opening of conductance channels in the sample and leads just like in Sec. III A. However, extending the investigation into the leads uncovers interesting behaviour in the current flow and curl. In Fig. 11, we see that the current flow reconstricts along the length of the leads after leaving the constriction ${ }^{32}$. This effect can be viewed in a classical picture with the electrons emerging from the constriction with some momentum $\mathrm{k}$ and repeatedly scattering off the walls of the lead in a ballistic fashion.

In Fig. 12, we can see the bouncy pattern behaviour which was observed in the $90^{\circ} \mathrm{S}$-bend. At this energy, there is only one conductance channel open in the sample. At first glance, it seems that the bouncy pattern cannot form without at least another channel open. However, the fifth conductance channel is opening in the leads. The mode travelling through the constriction transform into modes open in the lead. The modes in the leads mix to give the bouncy pattern. Both the bouncy patterns and the reconstricting current flows are quasi-periodic along the length of the lead. In Fig. 13, we can see these effects repeating up to a length of at least $300 \AA$. The repeated appearance of these two effects extends over lengths less than the inelastic mean free path for metals, of the order of 50 lattice parameters, at the melting temperature, in the bulk ${ }^{33}$. Beyond such lengths, phase breaking 

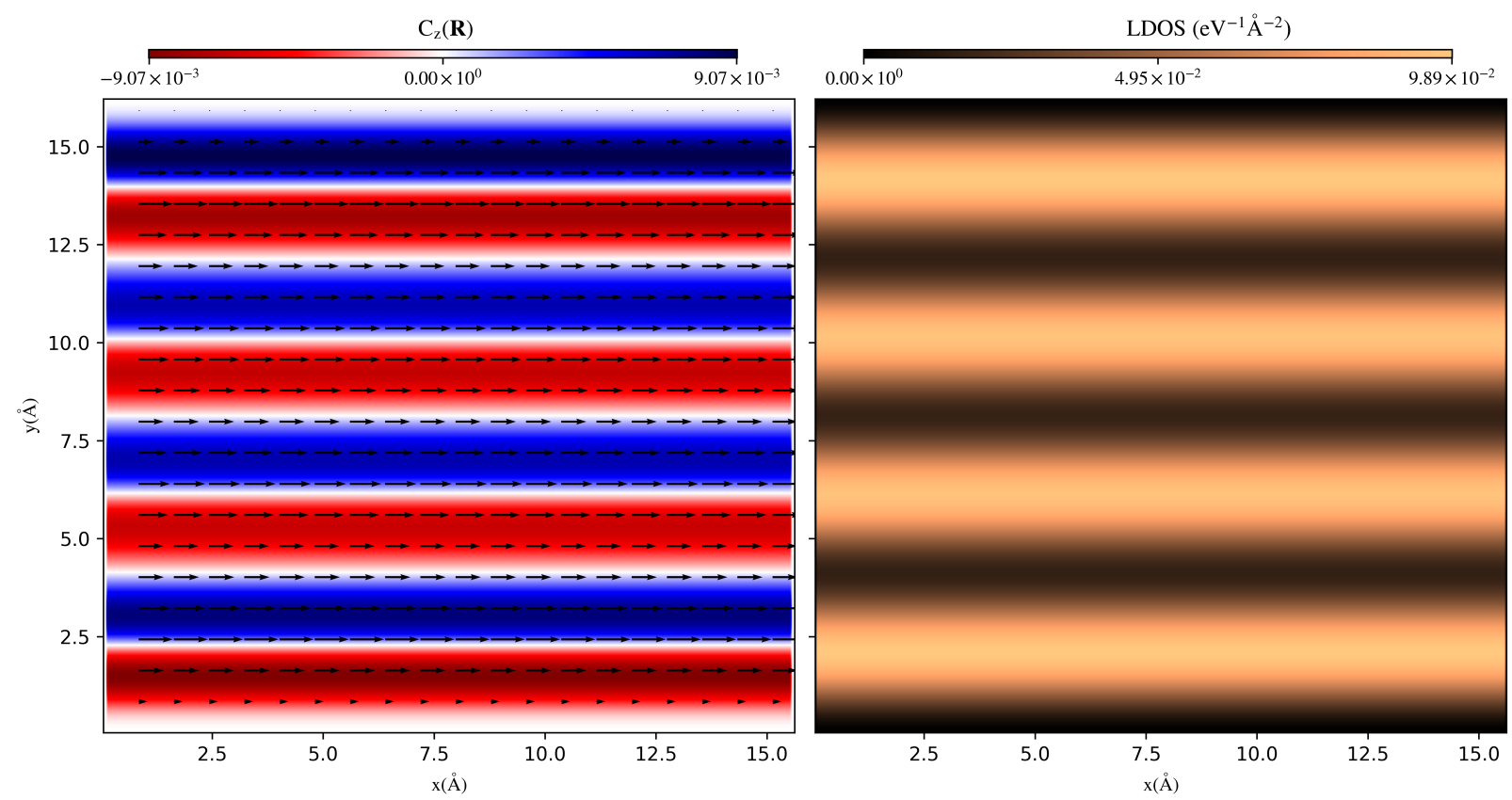

FIG. 7. Perfect wire: Plot of curl of the EWF in units of $2 \pi e \mathrm{~W} m \zeta^{2} /\left(\hbar^{2} \mathrm{eV} \AA^{4}\right)$ at $E_{F}=2.28 \mathrm{eV}$. The length of the arrows represents the magnitude of the current density. The background color, black to copper, represents the LDOS. At this energy another mode is opening up in the wire. Standing waves form in the LDOS giving rise to these large curls. Along the edges we can see even larger curls than the interior of the wire as the LDOS and current drop to zero. 

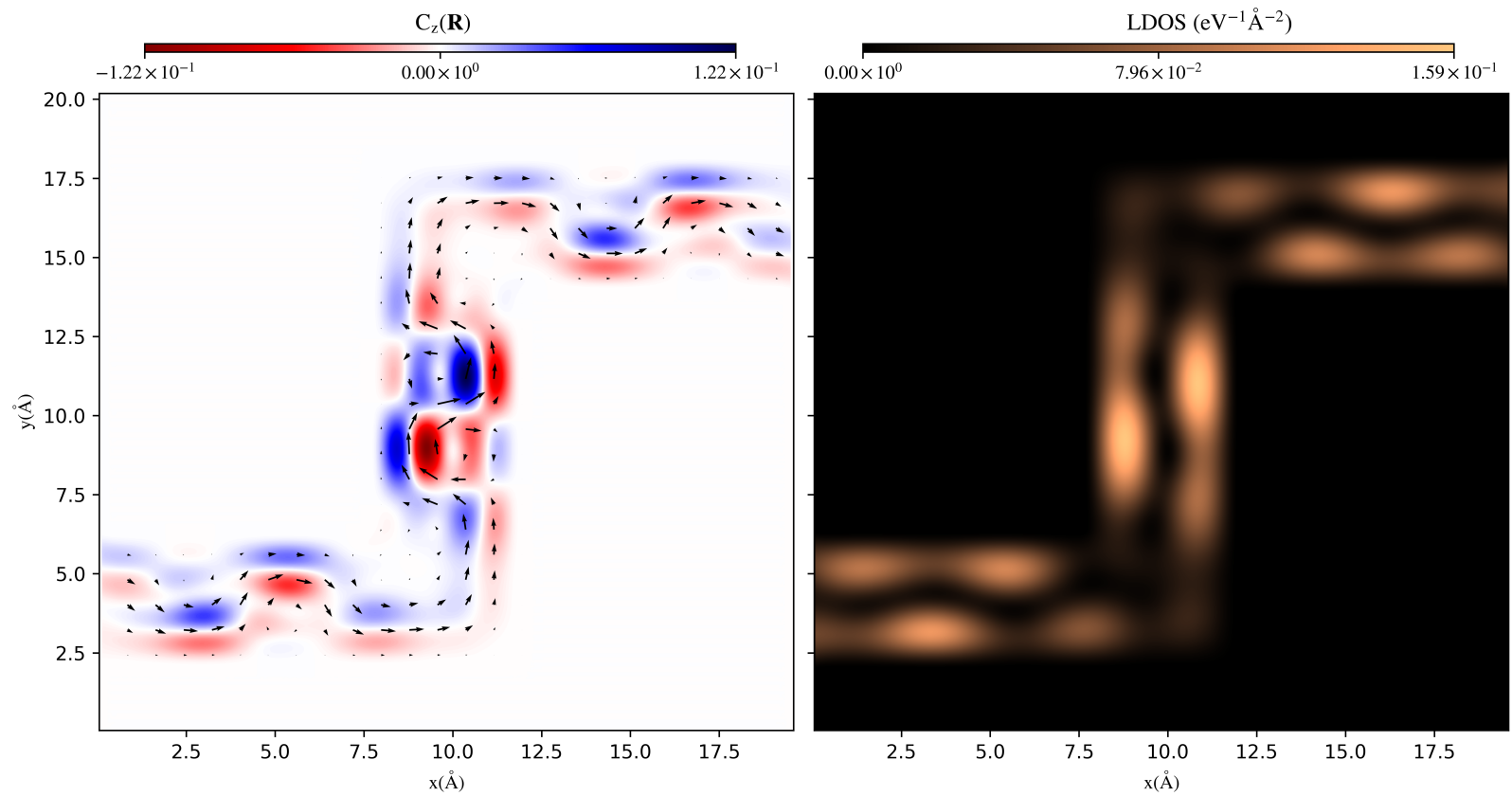

FIG. 8. $90^{\circ}$ S-bend: Plot of curl of the EWF in units of $2 \pi e \mathrm{Wm} \zeta^{2} /\left(\hbar^{2} \mathrm{eV} \AA^{4}\right)$ with $E=8.16 \mathrm{eV}$ and conductance $0.97\left(2 e^{2} / h\right)$. The length of the arrows represents the magnitude of the current density. At this energy, there are two channels open that can mix to form the bouncy pattern. The current 'snakes' along long sections of the wire, the changing direction results in a non-zero curl. The standing waves in the LDOS help amplify the curl. A lone pair current vortex is also evident in the middle section of the wire. 

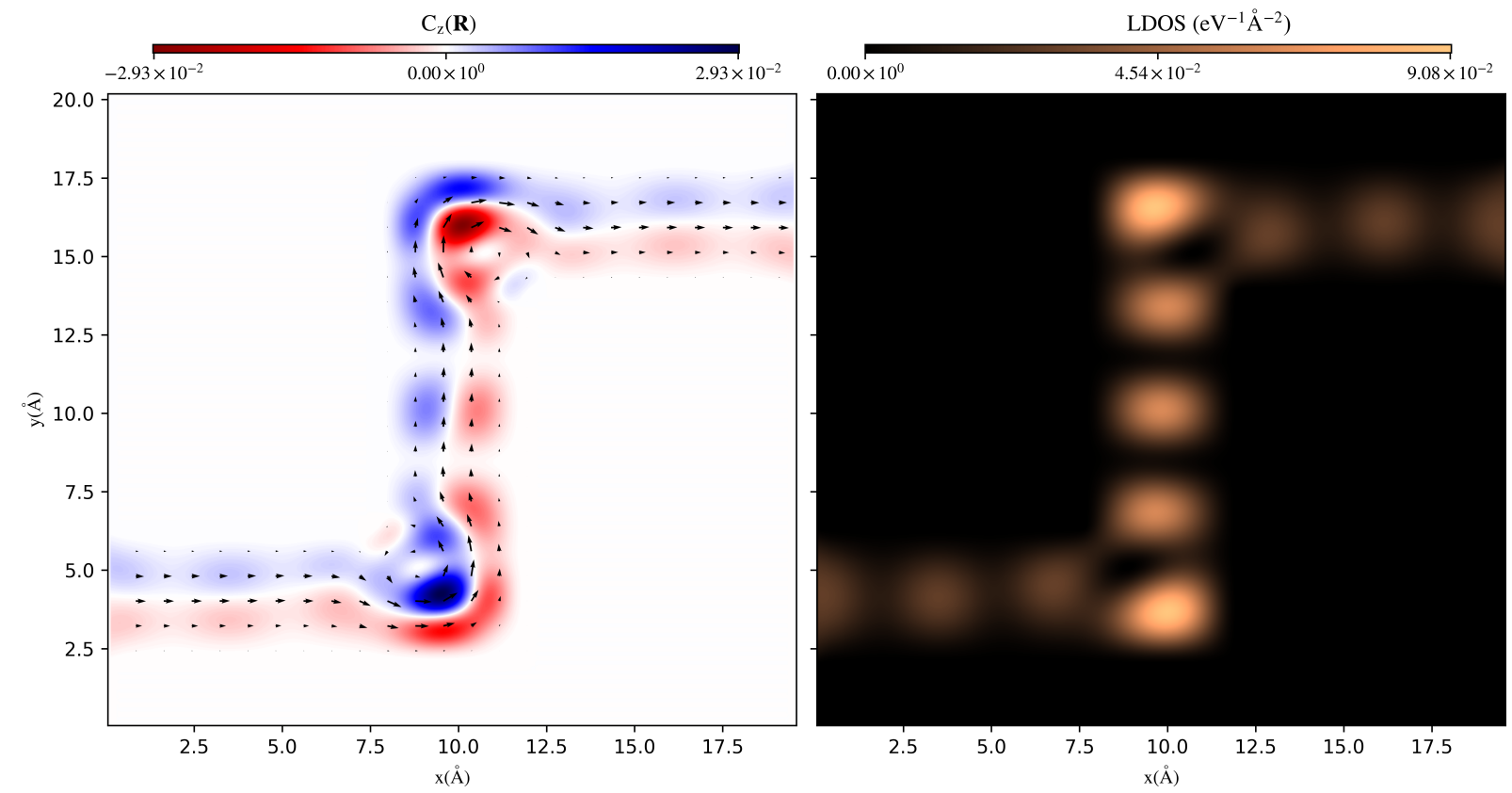

FIG. 9. $90^{\circ}$ S-bend: plot of curl of the EWF in units of $2 \pi e \mathrm{Wm} \zeta^{2} /\left(\hbar^{2} \mathrm{eV} \AA^{4}\right)$ with $E=5.44 \mathrm{eV}$ and conductance $0.84\left(2 e^{2} / h\right)$. The length of the arrows represents the magnitude of the current density. The curl at this energy is particularly high due to the presence of the current vortex. The presence of the lobe of high LDOS in the corners amplifies the curl in these regions. 

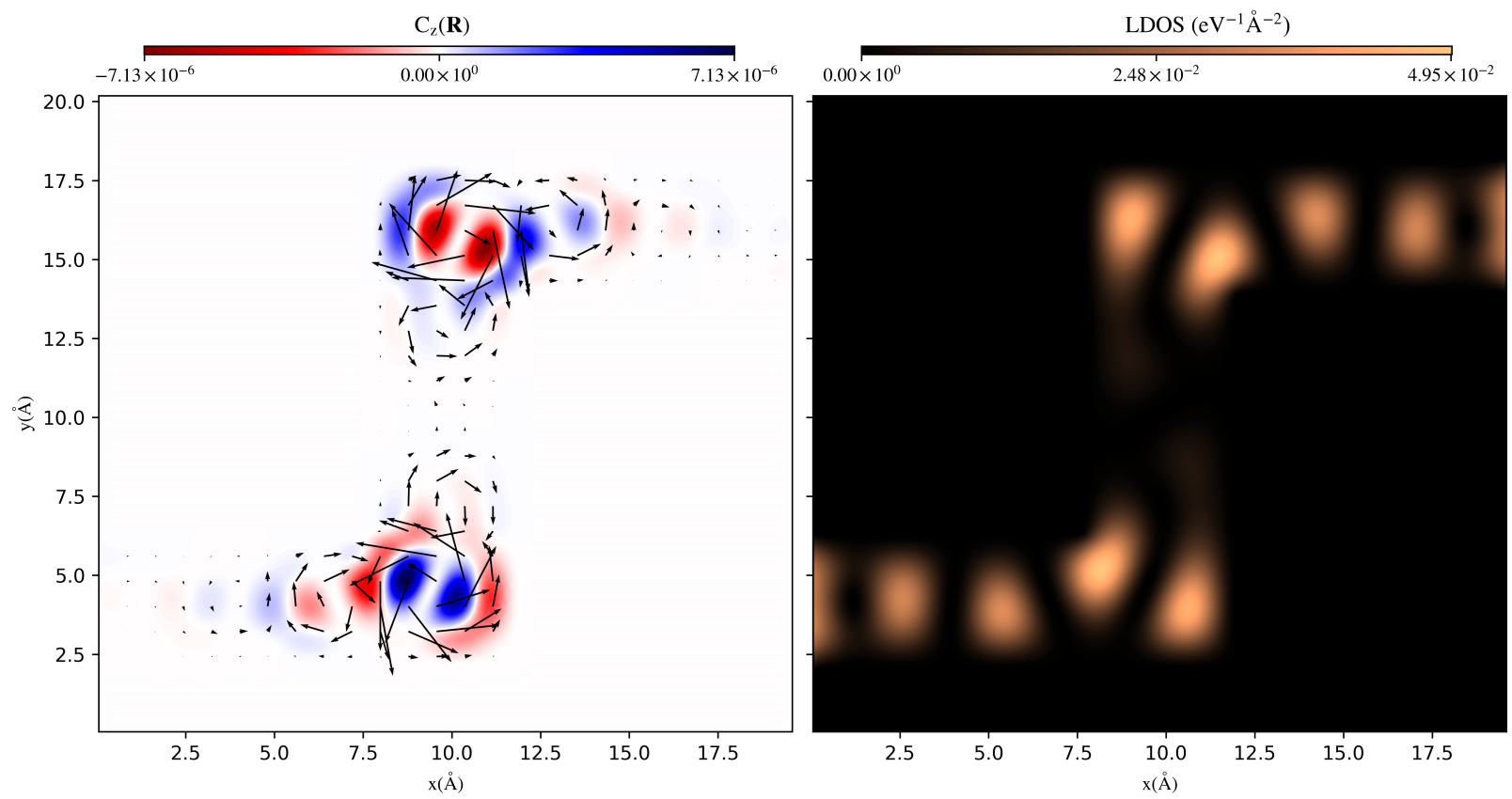

FIG. 10. $90^{\circ}$ S-bend: Plot of curl of the EWF in units of $2 \pi e \mathrm{Wm} \zeta^{2} /\left(\hbar^{2} \mathrm{eV} \AA^{4}\right)$ with $E=6.83 \mathrm{eV}$ and conductance $1.55 \times 10^{-5}\left(2 e^{2} / h\right)$. The length of the arrows represents the magnitude of the current density. Despite the presence of 8 current vortices and large internal currents relative to the net current, the curl is orders of magnitude lower than in Fig. 9. This low curl is due in part to the small internal currents relative to Fig. 9 and lower LDOS. 


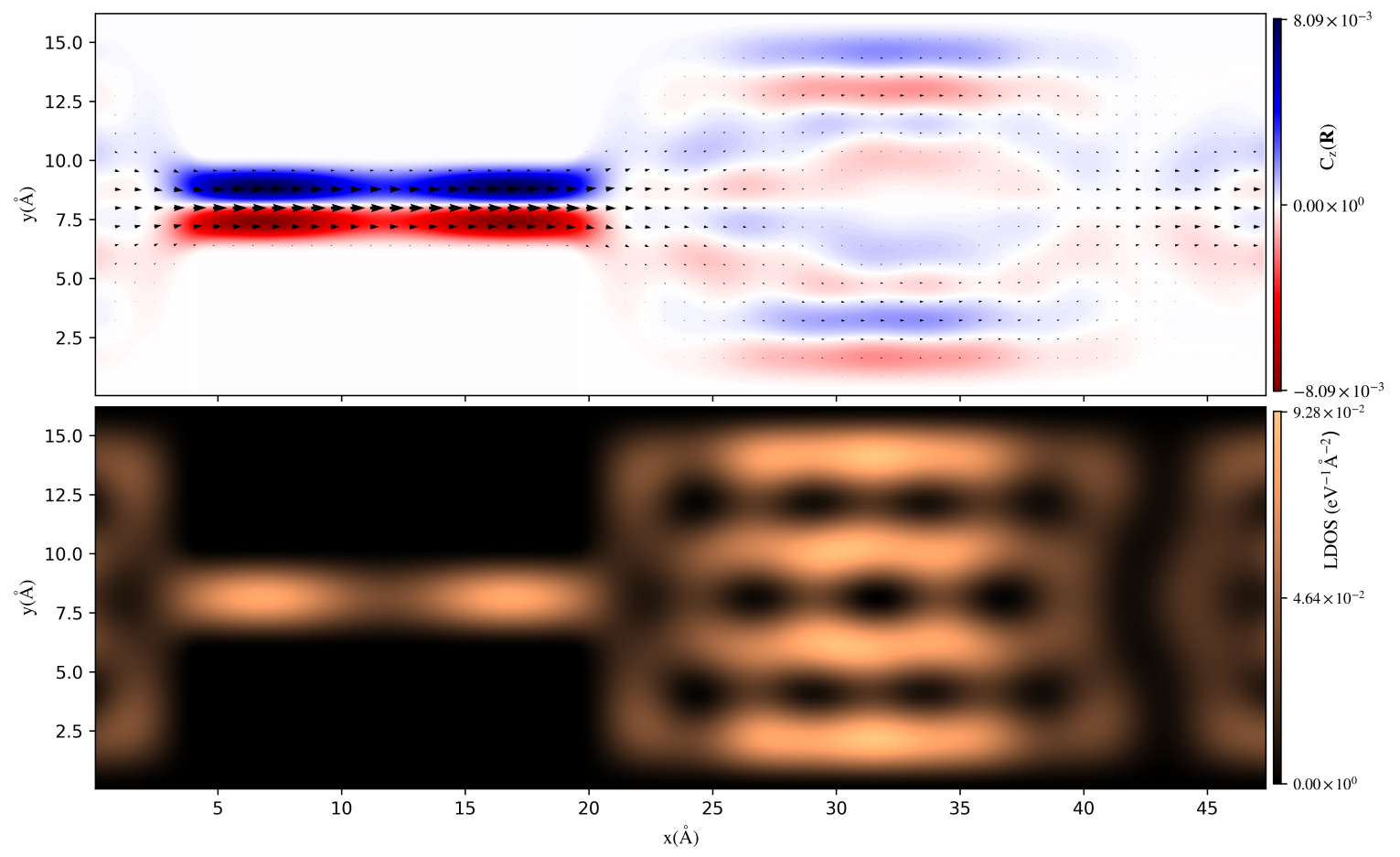

FIG. 11. Constriction: Plot of curl of the EWF in units of $2 \pi e \mathrm{~W} m \zeta^{2} /\left(\hbar^{2} \mathrm{eV} \AA^{4}\right)$ with $E=2.34 \mathrm{eV}$ and conductance $0.75\left(2 e^{2} / h\right)$. The length of the arrows represents the magnitude of the current density. The background color, black to copper, represents the LDOS. The flow reconstricts after it passes the constriction in the wire from ballistic collisions with the walls of the leads. This reconstriction repeats itself along the length of the wire. 


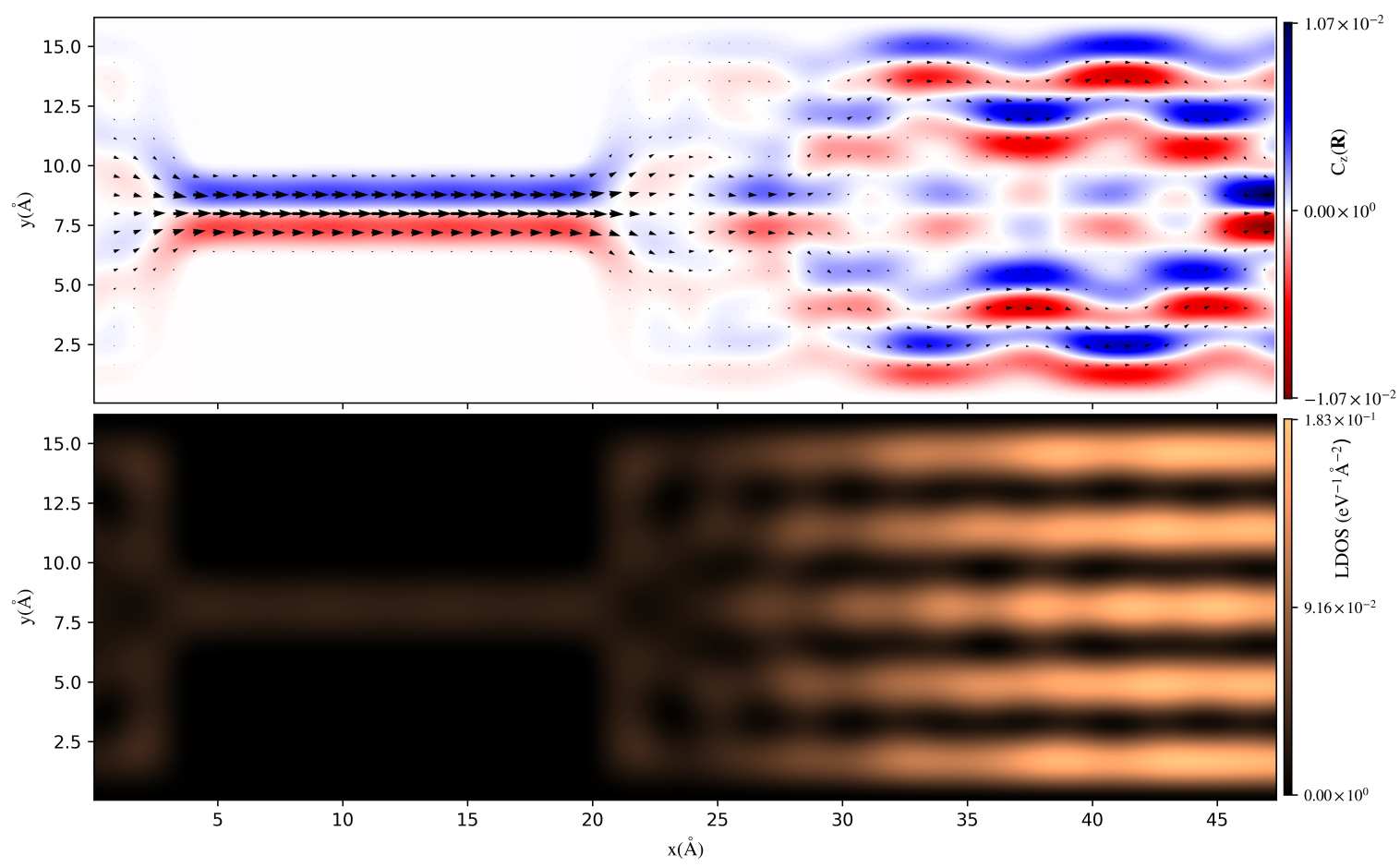

FIG. 12. Constriction: Plot of curl of the EWF in units of $2 \pi e \mathrm{~W} m \zeta^{2} /\left(\hbar^{2} \mathrm{eV} \AA^{4}\right)$ with $E=3.56 \mathrm{eV}$ and conductance $0.98\left(2 e^{2} / h\right)$. The length of the arrows represents the magnitude of the current density. The background color, black to copper, represents the LDOS. The curls are just as significant in the leads as the constriction, owing to the bouncy pattern coupled with the formation of standing waves in the LDOS in the leads. At this energy, only one channel is open in the constriction but there are five channels open in the leads. The modes in the leads mix and give rise to the bouncy pattern effect. The effect is quasi-periodic and repeats along the length of the leads. 


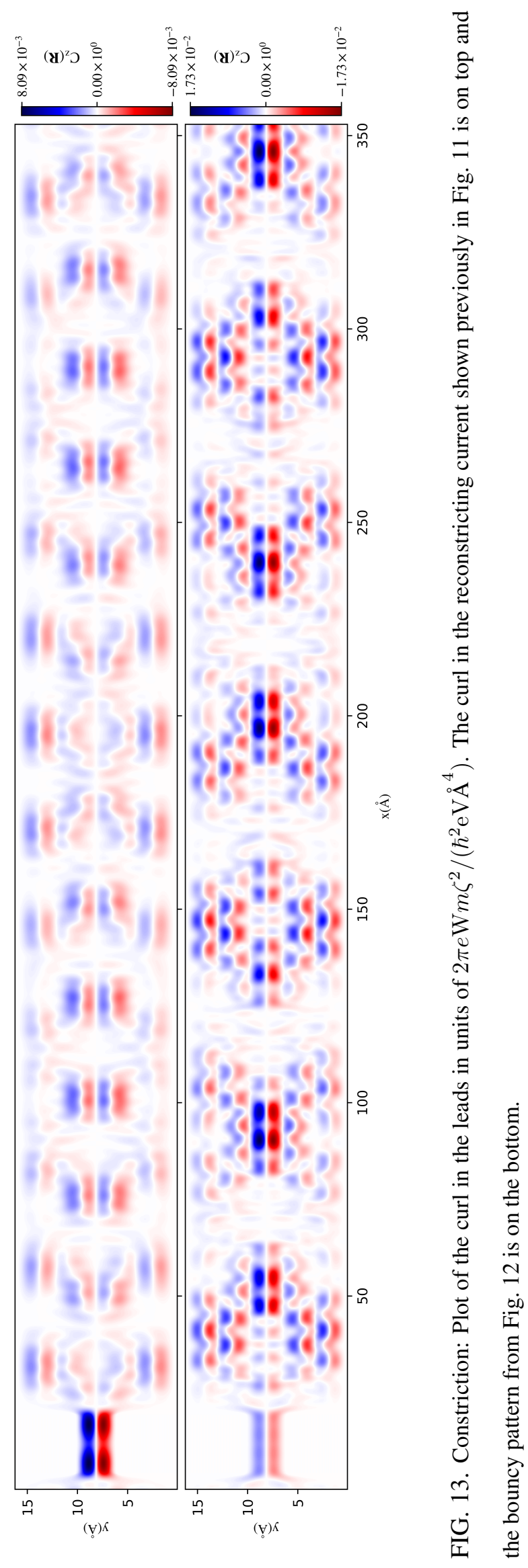


scattering ought to wash-out such behaviour in the curl patterns ${ }^{32}$. The reconstricting flows and bouncy patterns are potentially hazardous for the stability of the leads due to their ability to extend over long sections of a conductor.

\section{DISCUSSION}

The curl of the EWF has been calculated on a notional test scatterer up to this point. In a laboratory setting the test scatterer, for example, could represent an STM tip weakly coupled to the sample. An important feature of the curl maps that need to be investigated further is the physical size the regions of high curl need to be, for the nuclei to gain enough angular momentum to cause electromigration. Also, large positive curls often are seen in conjunction with a large negative curl nearby. In a dynamical simulation, they could, in theory, counteract each other with one curl doing work on a nucleus and the other draining it away. These problems require further work, with an explicit description of the dynamics ${ }^{10}$.

\section{CONCLUSIONS}

The curl of the EWF in the steady-state has been evaluated in the following systems: a perfect wire, a $90^{\circ} \mathrm{S}$-bend and a constriction. In perfect wires, at energies where a conductance channel opens, the curl within the wire becomes large. This is due to the variation and magnitude in the LDOS. The $90^{\circ} \mathrm{S}$-bend has demonstrated more complex behaviour. The bouncy pattern is particularly interesting as it leads to high curls that can extend over long segments of the wire. These bouncy patterns could significantly impact the structural integrity of a nanowire. We have also observed high- and low-curl current vortices. The occurrence of these vortices is potentially destructive and possibly a cause of electromigration. In the investigation of the simple constriction, we found exciting behaviour in the leads. We have observed the reconstriction of the current along the length of the leads. This effect can give rise to large curls in the leads at regions far away from the constriction. The bouncy patterns have also been observed and like the reconstricting current flow are quasi-periodic along the length of the leads, resulting in large curls in its wake.

It is hoped that the quantity studied in this paper - the curl of the EWF - will be of interest to other researchers, both experimentalists and theorists, as a way to map out regions where the nonconservative character of current-induced forces becomes important. Although for convenience 
our calculations assume a free-electron Hamiltonian, the method remains valid for an arbitrary one-electron potential. The strength of these effects in systems with varying levels of disorder is an interesting avenue for further work ${ }^{34}$. The influence of magnetic fields on the curl is also of interest and potentially opens up mechanisms to control it.

\section{ACKNOWLEDGMENTS}

We thank Mads Brandbyge and John Barker for helpful discussions, Malachy Montgomery for computational support and the anonymous referees for their constructive comments. We are grateful to EPSRC for funding this research (project reference EP/N509541/1 - 1941358).

*cmccooey02@qub.ac.uk

1 M. Lundstrom, Science 299, 210 (2003).

2 International Roadmap for Devices and Systems (2018).

3 N. Mingo and L. Yang and J. Han, J. Phys. Chem. B 105, 11142 (2001).

4 R. S. Sorbello, in Solid State Physics, Vol. 51, edited by H. Ehrenreich and F. Spaepen (Academic Press, 1997) pp. 159-231.

5 A. P. Horsfield, D. R. Bowler, A. J. Fisher, T. N. Todorov and M. J. Montgomery, J. Phys.: Condens. Matter 16, 3609 (2004).

6 J.-L. Lü, B.-Z. Hu, P. Hedegård and M. Brandbyge, Prog. Surf. Sci. 94, 21 (2019).

7 Z. Chen and R. S. Sorbello, Phys. Rev. B 47, 13527 (1993).

8 A. P. Horsfield, D. R. Bowler, H. Ness C. G. Sánchez, T. N. Todorov and A. J. Fisher, Rep. Prog. Phys. , 1195 (2006).

9 M. D. Ventra, Y. C. Chen, and T. N. Todorov, Phys. Rev. Lett. 92, 176803 (2004).

10 D. Dundas, E. J. McEniry, and T. N. Todorov, Nat. Nanotechnol. 4, 99 (2009).

11 J.-T. Lü, M. Brandbyge, and P. Hedegård, Nano Lett. 10, 1657 (2010).

12 T. N. Todorov, D. Dundas, J.-T. Lü, M. Brandbyge, and P. Hedegård, Eur J Phys 35, 065004 (2014).

13 R. B. Christensen and J.-T. Lü and P. Hedegård and M. Brandbyge, Beilstein J. of Nanotechnol. 7, 68 (2016).

14 R. Bustos-Marún, G. Refael, and F. von Oppen, Phys. Rev. Lett. 111, 060802 (2013). 
15 H. L. Calvo, F. D. Ribetto, and R. A. Bustos-Marún, Phys. Rev. B 96, 165309 (2017).

16 B. Cunningham and T. N. Todorov and D. Dundas, Beilstein J. Nanotechnol. 6, 2140 (2015).

17 N. Bode, S. V. Kusminskiy, R. Egger, and F. von Oppen, Phys. Rev. Lett. 107, 036804 (2011).

18 D. Dundas, B. Cunningham, C. Buchanan, A. Terasawa, A. T. Paxton, and T. N. Todorov, J. Phys.: Condens. Matter 24, 402203 (2012).

19 N. Agraï, A. L. Yeyati, and J. M. van Ruitenbeek, Phys. Rep. 377, 81 (2003).

20 M. Galperin, A. Nitzan, and M. A. Ratner, Phys. Rev. B 75, 19 (2007).

21 C. Sabster, C. Untiedt, and J. M. van Ruitenbeek, Beilstein J. Nanotechnol. 6, 2338 (2015).

22 T. N. Todorov, D. Dundas, A. T. Paxton, and A. P. Horsfield, Beilstein J. Nanotechnol. 2, 727 (2011).

23 M. J. Montgomery, T. N. Todorov, and A. P. Sutton, J. Phys.: Condens. Matter 14, 5377 (2002).

24 T. N. Todorov, J. Phys.: Condens. Matter 14, 3049 (2002).

25 A. T. Paxton, NIC Series 42, 145 (2009).

26 J. B. Pendry, A. Prêtre, P. J. Rous, and L. Martín-Moreno, Surf. Sci. 244, 160 (1991).

27 T. N. Todorov, Phys. Rev. B 54, 5801 (1996).

28 Z.-L. Ji, J. Appl. Phys. 73, 4468 (1993).

29 C. S. Lent, Appl. Phys. Lett. 56, 2554 (1990).

30 J. R. Barker, R. Akis, and D. K. Ferry, Superlat. Microstr. 27, 319 (2000).

31 J. R. Barker, Microelectron. Eng. 63, 223 (2002).

32 T. Can, H. Dai and D. K. Morr, Phys. Rev. B 85, 195459 (2012).

33 J. M. Ziman, in Principles of the Theory of Solids (Cambridge University Press).

34 R. B. S. Oakeshott and A. MacKinnon, J. Phys.: Condens. Matter 6, 1513 (1994).

\section{Appendix A: Convergence}

The investigation into the convergence involved the following mesh sizes: $a_{n}=a_{0} / 2^{n}$ where $a_{0}=0.397 \AA$ and $n=0,1,2,3$. A mesh size of $a=0.053 \AA$ was chosen to balance the tradeoff between computational cost and accuracy. While all calculations are performed in atomic units, we have listed the parameters in SI units. A plot of the conductance for the $90^{\circ} \mathrm{S}$-bend and constriction is shown in Fig. 14 and Fig. 15. The conductance quickly converges with mesh size. However, at energies where quasi-bound states are formed, convergence of the conductance is difficult to achieve. Take, for example, the sharp peaks in Fig. 14. Zooming in on the peaks 


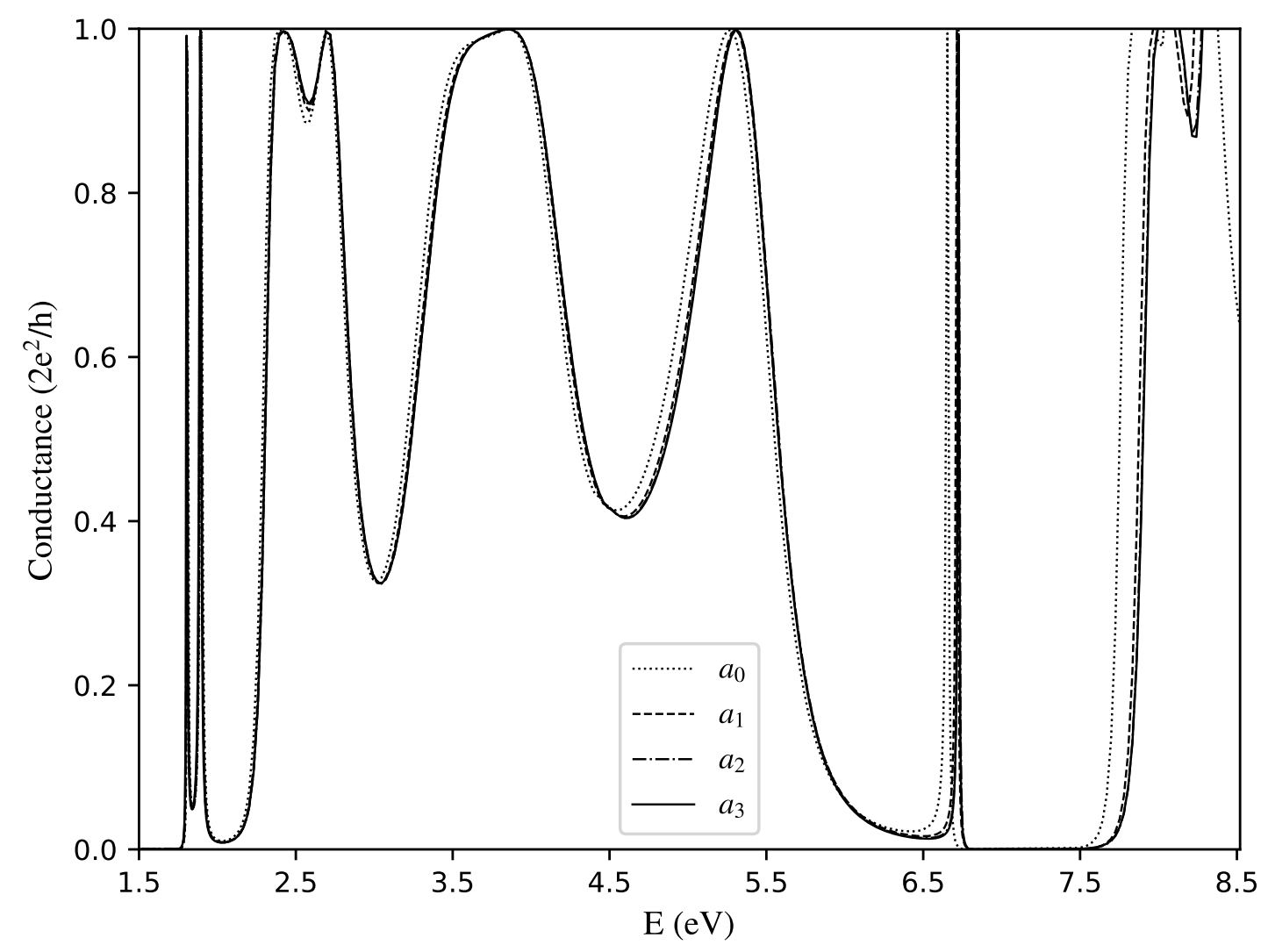

FIG. 14. $90^{\circ} \mathrm{S}$-bend: Plot of conductance and energy. The sharp peaks in the conductance are examples of resonances.

reveals that they are offset ever so slightly in energy. Due to the sharpness of the peaks, this energy offset gives significant disagreement in the conductance. These energies are close to a pole in the Green's function ${ }^{26}$. The investigation of the convergence with mesh size was extended to measure how the curl behaves spatially, both in a quantitative and qualitative sense.

The convergence of $L_{a}(E)$ is slow, both for $90^{\circ}$ S-bend shown in Fig. 16, and constriction shown in Fig. 17, but a definite trend demonstrating convergence can be seen as the mesh size reduces. A visual comparison was used to ascertain convergence at energies where particularly poor values of $L_{a}(E)$ were calculated. These poor values of $L_{a}(E)$ constitute small regions in the curl that cause the denominator in Eqn. 17 to tend to zero, giving a singularity and thereby masking an overall converged result. However, as the mesh size diminishes, it can still be difficult to achieve low values with $L_{a}(E)$. An example of this can be seen in Fig. 18 at an energy with a large $L_{a}(E)$. 


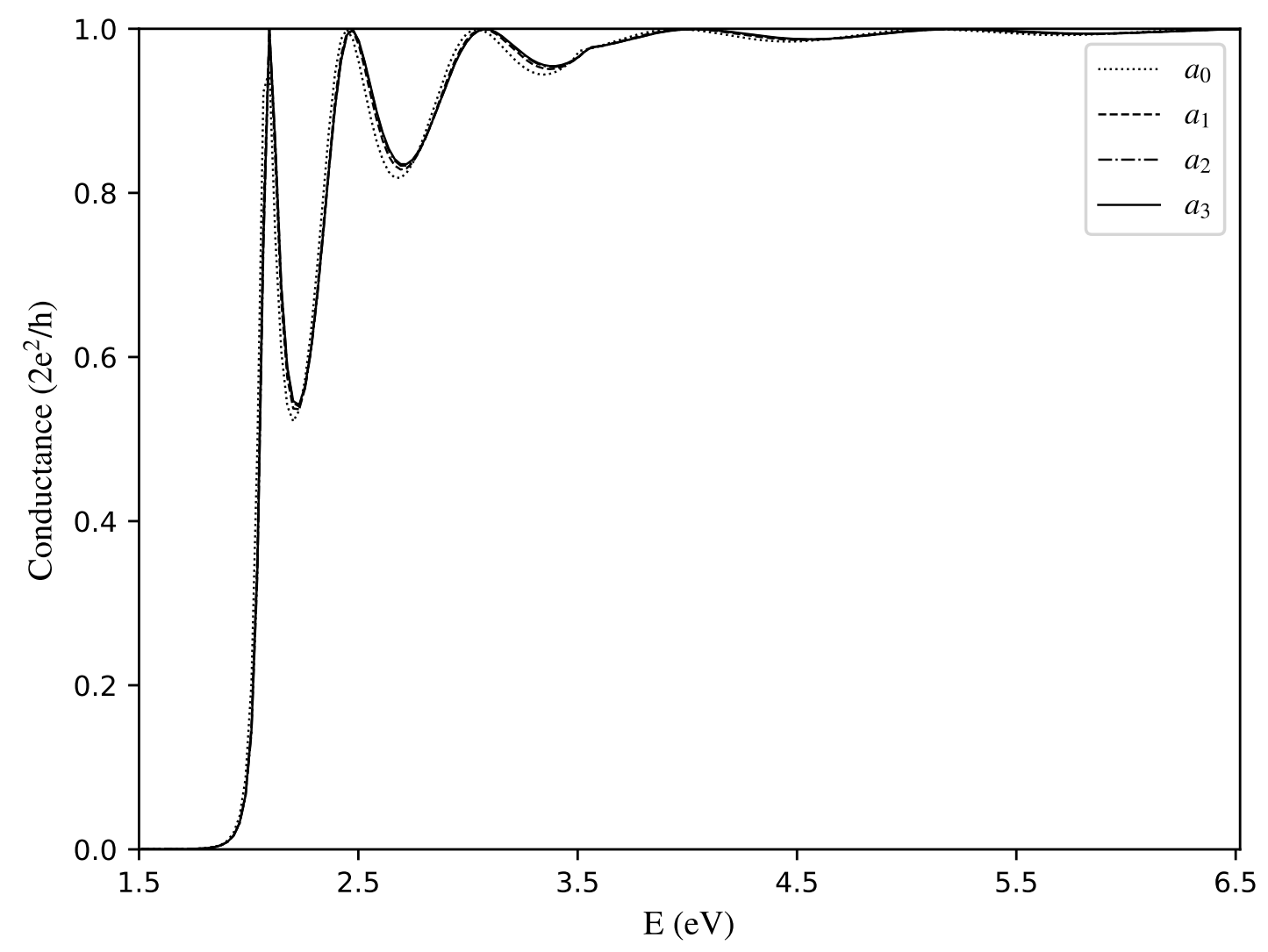

FIG. 15. Constriction: Plot of conductance and energy.

Here, we find that the patterns in the curl match up, but there is a significant disagreement in the magnitude of the curl between the two meshes. This behaviour occurs in the anti-resonance just above $6.8 \mathrm{eV}$ for the $90^{\circ} \mathrm{S}$-bend for example. There, the poor convergence of the curl comes not from the denominator but from the numerator in Eqn. 17.

A similar comparison between mesh sizes was made for the LDOS using Eqn. 17. The LDOS was found to converge quicker in comparison to the curl, in part due to the LDOS coming directly from the calculation of the Green's function and having a lower bound of zero. The convergence of the curl and LDOS at quasi-bound states, like the conductance, is poor. The convergence can be improved by increasing the value of $\epsilon$. However, the system no longer conserves current with a large $\epsilon$. 


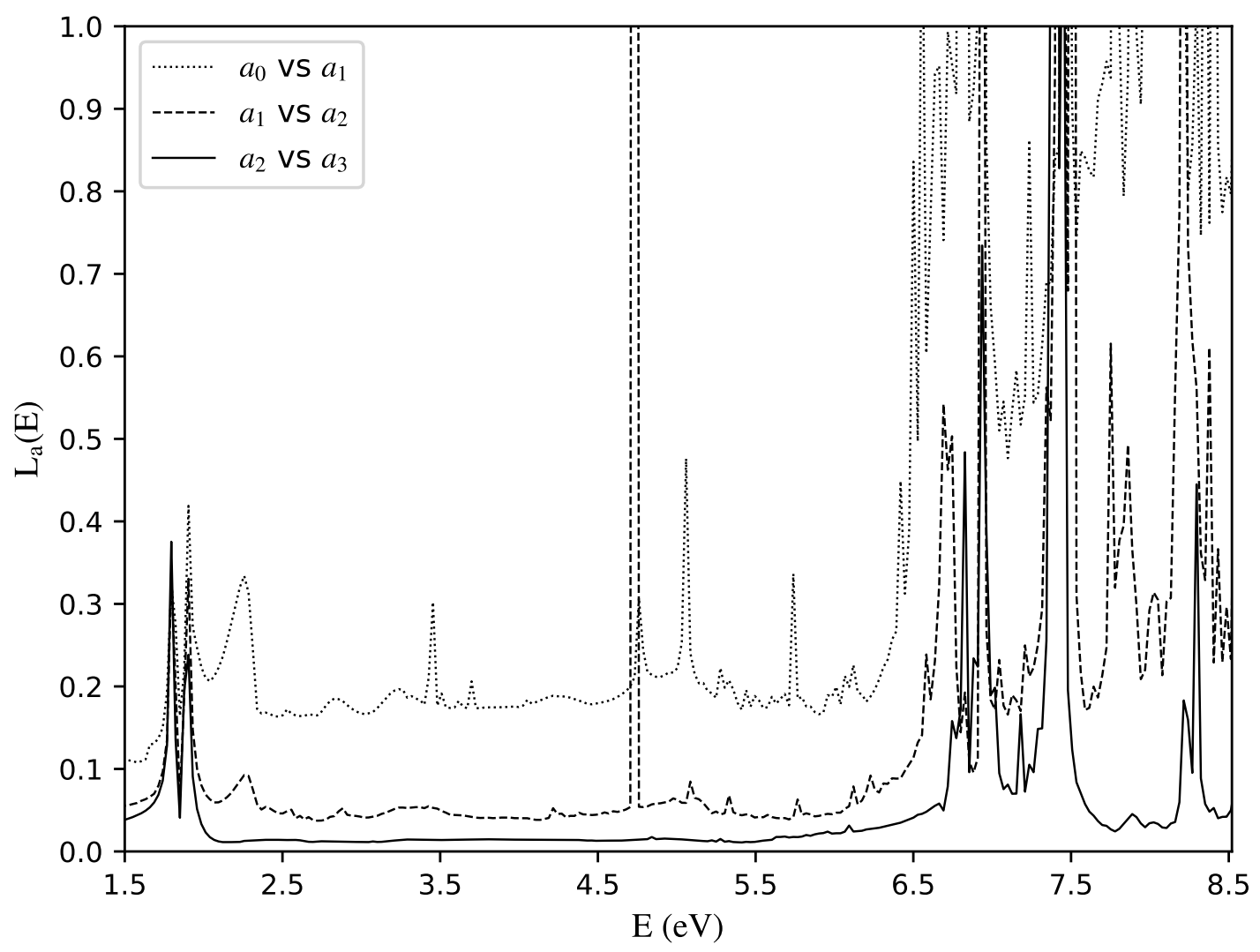

FIG. 16. $90^{\circ} \mathrm{S}$-bend: At first glance, it appears that the convergence of the sample is very poor. However, this is mainly due to the definition of $L_{a}(E)$. Visually comparing the curl reveals that the meshes start to converge nicely.

\section{Appendix B: Bouncy Patterns}

We give an argument for the necessary conditions for the bouncy pattern to occur. The bouncy patterns have a $j_{y}$ component that varies between positive and negative along the $x$-direction. The wavefunction of the electron in the wire can be expressed as a superposition of $\eta$ orthogonal wave components

$$
\psi=\sum_{i=1}^{\eta} a_{i} \phi_{i}(y) \exp \left(\mathrm{i} k_{i} x\right)
$$

where $\exp \left(\mathrm{i} k_{i} x\right), \phi_{i}(y)$ and $a_{i}$ is the right travelling wave, the transverse mode and amplitude respectively. Setting $\hbar=m=e=1$, the current density is given by

$$
\mathbf{J}=\frac{1}{2 \mathrm{i}}\left(\psi^{*} \nabla \psi-\psi \nabla \psi^{*}\right)
$$




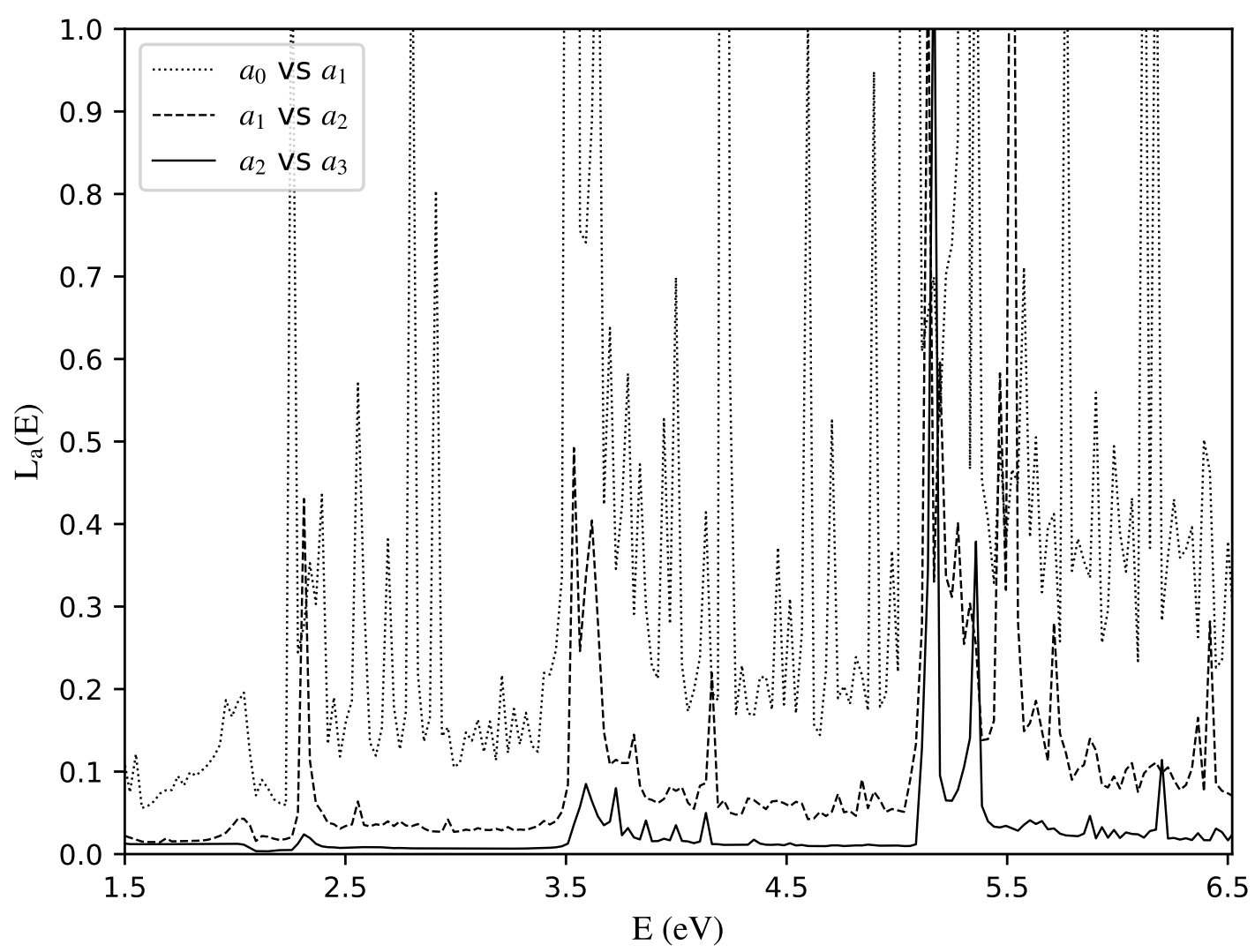

FIG. 17. Constriction: The convergence of the constriction is quicker than $90^{\circ} \mathrm{S}$-bend.

Considering only the y-component and expressing Eqn. B2 in terms of the real and imaginary part of $\psi$ we have

$$
J_{y}=\operatorname{Re}[\psi] \partial_{y} \operatorname{Im}[\psi]-\operatorname{Im}[\psi] \partial_{y} \operatorname{Re}[\psi]
$$

Substituting Eqn. B1 into Eqn. B3 gives

$$
\begin{aligned}
J_{y} & =\sum_{i=1}^{\eta} a_{i} \phi_{i}(y) \cos \left(k_{i} x\right) \sum_{j=1}^{\eta} a_{j} \phi_{j}^{\prime}(y) \sin \left(k_{j} x\right) \\
& -\sum_{i=1}^{\eta} a_{i} \phi_{i}(y) \sin \left(k_{i} x\right) \sum_{j=1}^{\eta} a_{j} \phi_{j}^{\prime}(y) \cos \left(k_{j} x\right) .
\end{aligned}
$$

Letting $a_{i} \phi_{i}(y)=A_{i}, a_{i} \phi_{i}^{\prime}(y)=B_{i}, C_{i}=\cos \left(k_{i} x\right)$, and $S_{i}=\sin \left(k_{i} x\right)$, Eqn. B4 can be expressed as

$$
J_{y}=\sum_{i=1}^{\eta} \sum_{j=1}^{\eta} A_{i} C_{i} B_{j} S_{j}-\sum_{i=1}^{\eta} \sum_{j=1}^{\eta} A_{i} S_{i} B_{j} C_{j}
$$



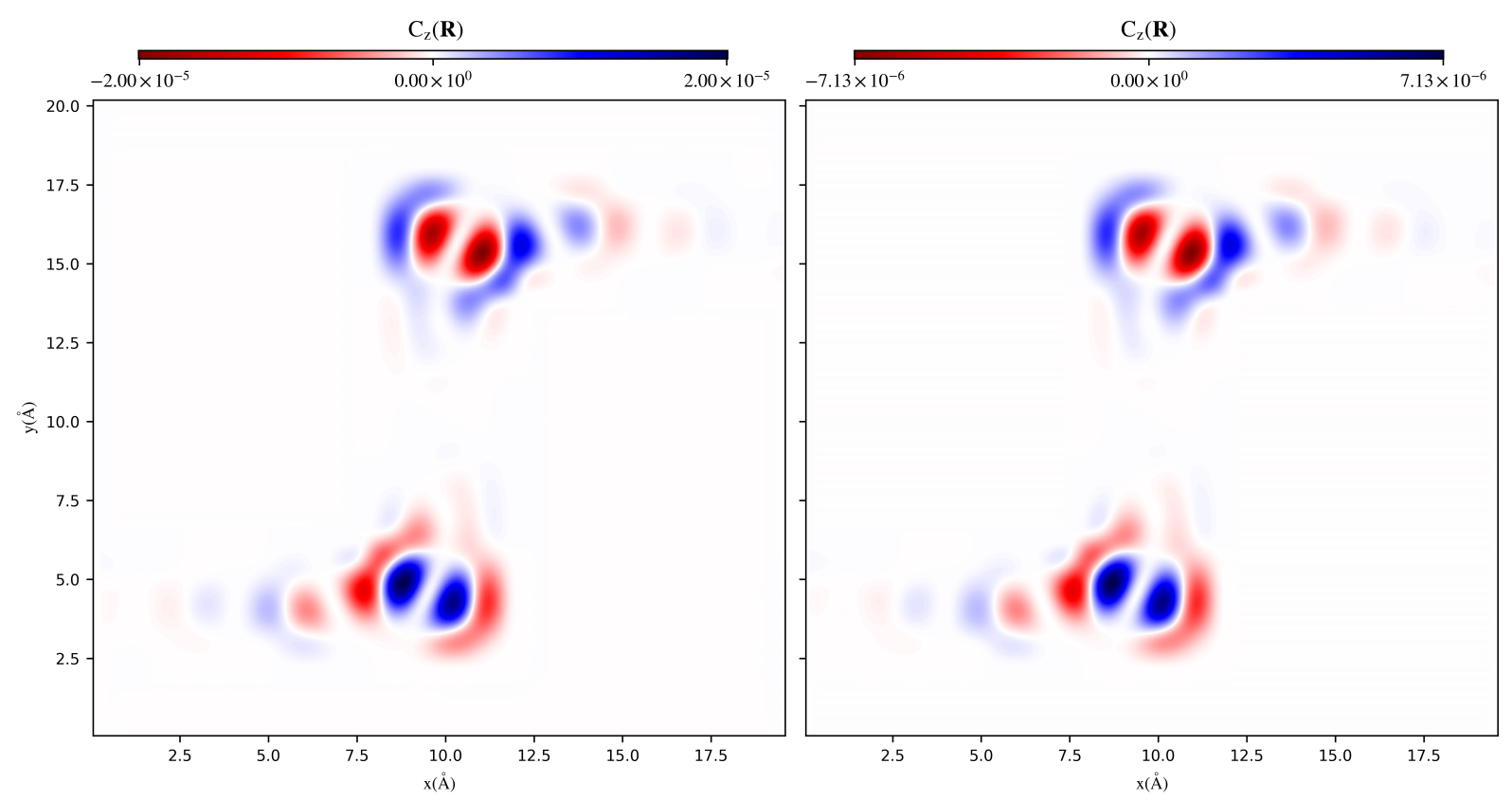

FIG. 18. $90^{\circ}$ S-bend: Comparison between the curl, in units of $2 \pi e \mathrm{~W} m \zeta^{2} /\left(\hbar^{2} \mathrm{eV} \AA^{4}\right)$, at $E_{F}=6.83 \mathrm{eV}$ of $a_{2}$ and $a_{3}$ respectively at a value with a very large percentage difference. Despite the large value of $L_{a}(E)$, there is good agreement matching the patterns in the curl between the two meshes. The large value of $L_{a}(E)$ arises from the difference in magnitude between the two plots.

Collecting terms together we have

$$
J_{y}=\sum_{i=1}^{\eta} \sum_{j=1}^{\eta} A_{i} B_{j}\left(C_{i} S_{j}-S_{i} C_{j}\right)
$$

Eqn. B6 can be written as

$$
J_{y}=\sum_{i=1}^{\eta} \sum_{j=1}^{\eta} A_{i} B_{j} \sin \left(\left(k_{i}-k_{j}\right) x\right) .
$$

Swapping $i$ and $j$ and adding the RHS to both sides Eqn. B7 gives

$$
2 J_{y}=\sum_{i=1}^{\eta} \sum_{j=1}^{\eta} A_{i} B_{j} \sin \left(\left(k_{i}-k_{j}\right) x\right)+A_{j} B_{i} \sin \left(\left(k_{j}-k_{i}\right) x\right)
$$

which can written as

$$
J_{y}=\sum_{i=1}^{\eta} \sum_{j=1}^{\eta} \frac{1}{2}\left(A_{i} B_{j}-A_{j} B_{i}\right) \sin \left(\left(k_{i}-k_{j}\right) x\right) .
$$

Letting $D_{i j}=-D_{j i}=\frac{1}{2}\left(A_{i} B_{j}-A_{j} B_{i}\right)$ we have

$$
J_{y}=\sum_{i=1}^{\eta} \sum_{j=1}^{\eta} D_{i j}(y) \sin \left(\left(k_{i}-k_{j}\right) x\right) .
$$


Inspecting $J_{y}$ for $\eta=1$ we can see that $J_{y}$ is zero. Therefore, at least two modes are needed for a bouncy pattern to form. The periodicity of the bouncy pattern emerges from the sine terms in Eqn. B10. 